\title{
Evolutionary conservation of the U7 small nuclear ribonucleoprotein in Drosophila melanogaster
}

\author{
TELDJA N. AZZOUZ and DANIEL SCHÜMPERLI \\ Institute of Cell Biology, University of Bern, 3012 Bern, Switzerland
}

\begin{abstract}
The U7 snRNP involved in histone RNA $3^{\prime}$ end processing is related to but biochemically distinct from spliceosomal snRNPs. In vertebrates, the $\mathrm{Sm}$ core structure assembling around the noncanonical Sm-binding sequence of U7 snRNA contains only five of the seven standard Sm proteins. The missing Sm D1 and D2 subunits are replaced by U7-specific Sm-like proteins Lsm10 and Lsm11, at least the latter of which is important for histone RNA processing. So far, it was unknown if this special U7 snRNP composition is conserved in invertebrates. Here we describe several putative invertebrate Lsm10 and Lsm11 orthologs that display low but clear sequence similarity to their vertebrate counterparts. Immunoprecipitation studies in Drosophila S2 cells indicate that the Drosophila Lsm10 and Lsm11 orthologs (dLsm10 and dLsm11) associate with each other and with Sm B, but not with Sm D1 and D2. Moreover, dLsm11 associates with the recently characterized Drosophila U7 snRNA and, indirectly, with histone H3 pre-mRNA. Furthermore, dLsm10 and dLsm11 can assemble into U7 snRNPs in mammalian cells. These experiments demonstrate a strong evolutionary conservation of the unique U7 snRNP composition, despite a high degree of primary sequence divergence of its constituents. Therefore, Drosophila appears to be a suitable system for further genetic studies of the cell biology of U7 snRNPs.
\end{abstract}

Keywords: Small nuclear ribonucleoprotein; histone pre-mRNA 3' processing; Sm-like protein; Sm core structure; Drosophila melanogaster

\section{INTRODUCTION}

The formation of nonpolyadenylated histone mRNA 3' ends in metazoans is the only RNA processing reaction known to be devoted entirely to the expression of a specific protein family (for review, see Müller and Schümperli 1997; Dominski and Marzluff 1999; Marzluff and Duronio 2002). This special RNA maturation pathway is intimately linked to the cell cycle regulation of histone gene expression (Harris et al. 1991). However, plant and fungal histone mRNAs, which are also cell cycle-regulated, are polyadenylated and appear to be generated by the same RNA processing pathway used by other protein-coding genes of these organisms.

Although it shares the need for an Sm-type snRNP with pre-mRNA splicing and certain biochemical features with the $3^{\prime}$ end cleavage/polyadenylation reaction (for discussion, see Müller and Schümperli 1997), histone RNA processing is biochemically distinct from both of these RNA

Reprint requests to: Daniel Schümperli, Institute of Cell Biology, University of Bern, Baltzerstrasse 4, 3012 Bern, Switzerland; e-mail: daniel.schuemperli@izb.unibe.ch; fax: 41-31-6314616.

Article and publication are at http://www.rnajournal.org/cgi/doi/ 10.1261/rna.5143303. maturation pathways. Several trans-acting factors for histone RNA processing have been identified and studied, primarily in vertebrate systems.

1. The minor U7 snRNP binds to the purine-rich histone downstream element (HDE; Schaufele et al. 1986; Bond et al. 1991) and thereby serves as a molecular ruler, positioning the yet unknown cleavage activity near the processing site (Scharl and Steitz 1994, 1996). U7 snRNA is 58-71 nucleotides long, depending on the species, and like spliceosomal snRNAs, it bears a trimethyl guanosine cap at its $5^{\prime}$ end. Following the $5^{\prime}$ region complementary to the HDE, U7 snRNA contains a noncanonical Smbinding site (AAUUUGUCUAG in mammals), followed by a long hairpin structure and a very short singlestranded 3' extension.

2. The histone hairpin binding protein (HBP; Vasserot et al. 1989; Martin et al. 1997), also known as stem-loop binding protein (SLBP; Wang et al. 1996) binds to a highly conserved RNA hairpin just upstream of the cleavage site. This interaction contributes to efficient histone RNA processing by favoring the formation of the histone pre-mRNA/U7 snRNP complex (Streit et al. 1993; Spycher et al. 1994; Dominski et al. 1999). 
3. This effect of HBP on processing appears to be mediated by a $100-\mathrm{kD}$ zinc finger protein, ZFP100, that binds to the HBP/hairpin complex (Dominski et al. 2002a) and also interacts with the $\mathrm{N}$ terminus of the U7 snRNPspecific protein Lsm11 (Pillai et al. 2003; see below).

However, the details of the interactions between these known processing factors still require further investigation, and some processing factors may still await their discovery.

Our recent studies of the protein composition of vertebrate U7 snRNPs have led to the understanding that a special type of Sm core structure assembles around the Smbinding site of U7 snRNA. Whereas the Sm core of spliceosomal snRNPs contains the seven Sm proteins B/B', D1, D2, D3, E, F, and G (for review, see Kambach et al. 1999), the U7 Sm core has Lsm10 and Lsm11 replacing Sm D1 and D2 (Pillai et al. 2001, 2003). This unique Sm core composition has two important implications: First, Lsm11, through its long N-terminal extension, participates in histone RNA processing, and it may do so partly through its interaction with ZFP100 (Pillai et al. 2003; see above). Second, the cell must be able to discern between snRNAs with only slightly different Sm-binding sites, and form the required type of Sm core-spliceosomal or U7-specific-without making mistakes. It apparently does so by preforming specific assembly complexes containing the survival of motor neurons protein (SMN), several Gemin proteins, and either Sm D1/ D2 or Lsm10/11, in combination with the other Sm proteins (Pillai et al. 2003). After assembly, the U7 snRNP is imported into the nucleus, where it is highly enriched in Cajal bodies (Wu and Gall 1993; Frey and Matera 1995). Thus, the U7 snRNP not only is the key component and nucleation center of the histone RNA processing complex but also represents an important model for studying the processes of snRNP assembly, nuclear import, and Cajal body formation.

Both HBP and the hairpin are highly conserved and can be identified by sequence comparison in metazoans as well as in certain lower organisms such as Chlamydomonas and Volvox (Wittop Koning and Schümperli 1994; http://www. izb.unibe.ch/res/schuehome/schuemperli/hbppep.html). Moreover, HBP orthologs have been characterized biochemically in mammals, Xenopus, Drosophila, and Caenorhabditis elegans. In contrast, the HDE is conserved to some extent between vertebrates and echinoderms, but in lower invertebrates, the sequences downstream of histone mRNA $3^{\prime}$ ends are divergent, and an HDE is therefore difficult to identify (for review, see Wittop Koning and Schümperli 1994; Dominski and Marzluff 1999). Thus, until recently, HDEs or U7 snRNAs had not been defined in invertebrates other than sea urchins, and orthologs of Lsm10 or ZFP100 could not be identified by sequence similarity searches (Pillai et al. 2001; Dominski et al. 2002a). It was not clear if the inability to find these features was due to poor evolutionary conservation of primary sequences, or if histone RNA $3^{\prime}$ end processing in lower invertebrates operated by a mechanism independent of a U7 snRNP. However, by using an in vitro processing system from Drosophila tissue culture cells, Dominski et al. (2002b), characterized certain mutations downstream of a Drosophila histone RNA processing site that appeared to define a Drosophila HDE, and they also succeeded in characterizing a Drosophila U7 snRNA of 71 nucleotides (Dominski et al. 2003).

Here, we have taken a different approach to characterize the Drosophila U7 snRNP. The recently obtained sequence for the large Lsm11 protein (Pillai et al. 2003) has allowed us to detect potential invertebrate orthologs. Moreover, thanks to the recently published mosquito genome sequence and by using recursive sequence similarity searches, we have succeeded in detecting potential invertebrate Lsm10 orthologs as well. We have studied the interactions of the putative Drosophila Lsm10 and Lsm11 proteins (termed dLsm10 and dLsm11, respectively) with other Sm proteins, as well as with U7 snRNA in Drosophila tissue culture cells. Moreover, we demonstrate an indirect interaction of dLsm11 with histone pre-mRNA. Finally, dLsm10 and dLsm11 can assemble into U7 snRNPs in mammalian cells. All these data indicate that, despite considerable differences in primary sequences, the overall architecture of the U7 snRNP and the mechanism of histone RNA 3' end processing have been conserved over at least $\sim 1000 \mathrm{Myr}$, that is, the evolutionary time separating arthropods and vertebrates.

\section{RESULTS}

\section{Detection of potential invertebrate orthologs for Lsm10 and Lsm11}

Our Lsm10 database (http://www.izb.unibe.ch/res/ schuehome/schuemperli/Lsm10.html) currently comprises six complete mammalian sequences (human, mouse, rat, pig, bovine, and dog), as well as two amphibian (Xenopus laevis, Silurana tropicalis), one avian (chicken), and four fish sequences (fugu, medaka, rainbow trout, and zebrafish). By using the lower vertebrate sequences, we could identify potential orthologs from two tunicate species (Ciona intestinalis and Boltenia villosa). Additional iterative BLAST searches revealed a partial sequence from the mosquito Anopheles gambiae and a potential ortholog in Drosophila melanogaster. It is evident from the alignment of these invertebrate Lsm10 sequences with representative vertebrate ones (Fig. 1) that the vertebrate Lsm10 proteins are well conserved, but the sequences found in invertebrates are so divergent that the identification of the corresponding proteins as true Lsm10 orthologs, in the absence of biochemical evidence, remains questionable. However, it is also noteworthy that many of the amino acid changes are conservative in nature.

By using the same iterative search strategy, we found 


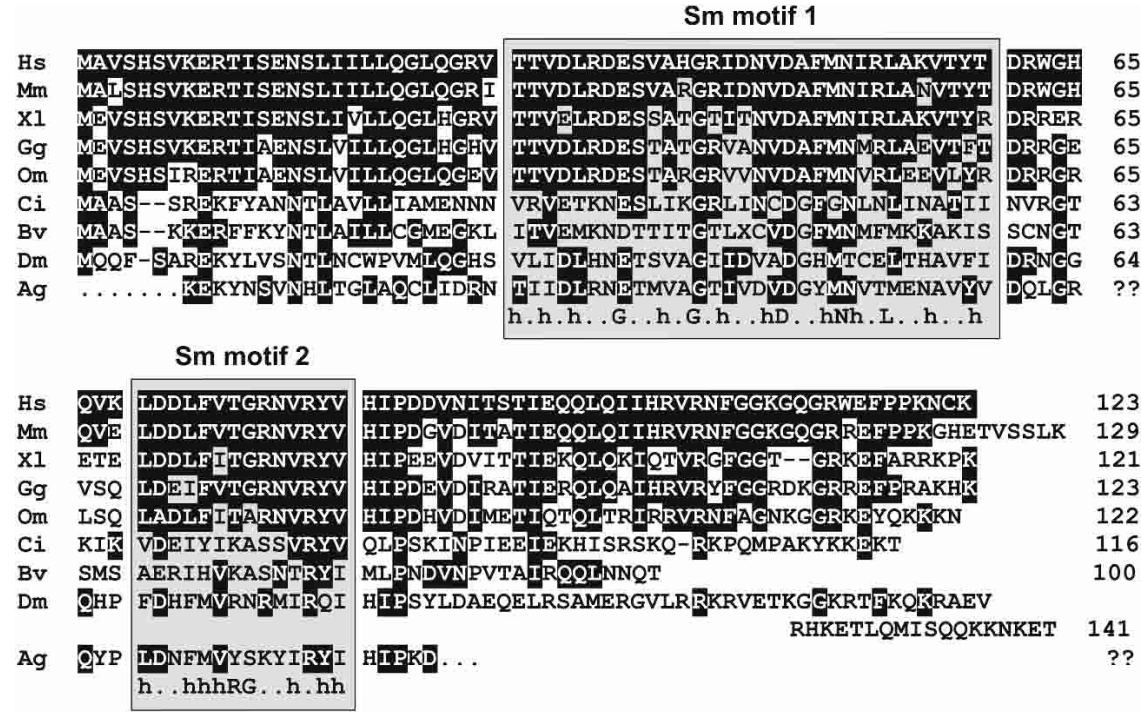

FIGURE 1. Sequence alignment of Lsm10 proteins. Additional sequences, accession numbers, and links are available at our online database (http://www.izb.unibe.ch/res/schuehome/ schuemperli/Lsm10.html). The sequences are from the following species: Hs, Homo sapiens, human (Unigene Hs.3496); Mm, Mus musculus, house mouse (Unigene Mm.41865); Xl, Xenopus laevis (Unigene Xl.6554); Gg, Gallus gallus, chicken (ESTs BU211735, BU445159, BU461367); Om, Oncorhynchus mykiss, rainbow trout (EST CA368590); Ci, Ciona intestinalis, transparent sea squirt (ascidian tunicate; Unigene Cin.8743); Bv, Boltenia villosa (ascidian tunicate; protein AAM76159); Dm, Drosophila melanogaster (FlyBase CG12938); and Ag, Anopheles gambiae, African malaria mosquito (predicted partial ORF EAA01310). Residues identical to the human protein are shown in inverse print. The conserved Sm motifs 1 and 2 are indicated by shaded boxes. The consensus sequences were adapted from previous sources (Hermann et al. 1995; Achsel et al. 2001). h indicates hydrophobic amino acids.

putative invertebrate Lsm11 orthologs in C. intestinalis and D. melanogaster (Fig. 2). Their detection was facilitated by the presence of a long $\mathrm{N}$-terminal extension and of an extensive spacer separating the two Sm motifs in Lsm11 (Pillai et al. 2003). The spacer is poorly conserved in sequence or length, but it is always longer than in any of the other $\mathrm{Sm} /$ Lsm proteins. Moreover, the $\mathrm{N}$-terminal extension contains several conserved sequence patches that can also be recognized in the invertebrate proteins (Fig. 2). An Lsm11 database containing additional sequences is maintained at our Web site (http://www.izb.unibe.ch/res/schuehome/ schuemperli/Lsm11.html).

\section{Drosophila Lsm10 and Lsm11 interact with each other and with $\mathrm{Sm}$ proteins}

To analyze whether the putative Drosophila orthologs dLsm10 and dLsm11 associate with each other and with Sm protein-containing complexes, we cloned the corresponding open reading frames (ORFs), each containing an HAtag at the $\mathrm{N}$ terminus, into an expression vector under the control of the Drosophila metallothionein promoter (see Materials and Methods). The HA-dLsm11 protein was well expressed after transient transfection in Drosophila S2 cells (Fig. 3A,B, input lanes). However, the expression of
HA-dLsm10 was much lower, so the protein was sometimes barely detectable by immunoblotting crude nuclear extract with anti-HA antibody (see input lane in Fig. 3B). However, importantly, HA-dLsm10 expressed in S2 cells could be coprecipitated by a polyclonal antibody raised against bacterially expressed, GST-tagged dLsm11 (Fig. 3A, lower panel). A control experiment showed that the same antibody also precipitated HA-dLsm11 (Fig. 3A, upper panel). Moreover, both proteins could be coprecipitated by the monoclonal anti-Sm antibody Y12 (Fig. 3B). These results indicated that $\mathrm{dLsm} 10$ and dLsm11 interact with each other as well as with some of the standard Sm proteins in Drosophila S2 cells.

The reactivity of these antibodies was tested by Western blots. By using antidLsm11 antibody, we could not detect a signal for the endogenous protein in S2 cells, presumably because of low expression of dLsm11 or insufficient antibody activity (Fig. 3C, left panel). Similarly, endogenous Lsm11 from human tissue culture cells could previously not be detected by immunoblotting with an antibody raised against human Lsm11, unless U7 snRNPs had been enriched by biochemical fractionation or by immunoprecipitation (Pillai et al. 2003). However, a signal was detectable with the anti-dLsm11 antibody in Drosophila S2 cells stably expressing HA-dLsm 11 from the metallothionein promoter, and this signal comigrated on gels with the band detected by anti-HA antibody (Fig. 3C). Taken together, these results proved that the antidLsm11 antibody reacts specifically with dLsm 11 .

Anti-Sm antibodies, in vertebrates, are known to react with the Sm proteins $\mathrm{B} / \mathrm{B}^{\prime}, \mathrm{D} 1$, and $\mathrm{D} 3$ (Lehmeier et al. 1990), which contain symmetrical dimethyl arginine modifications on their RG-rich C-terminal tails (Brahms et al. 2000, 2001). However, by using the Y12 antibody, we could only detect the $\mathrm{B} / \mathrm{B}^{\prime}$ and $\mathrm{D} 1$ proteins, not $\mathrm{D} 3$, on Western blots of nuclear extracts from human 293-T cells (Fig. 3D) or other mammalian cell lines (data not shown). Probing a Drosophila S2 cell nuclear extract on the same blot revealed two similar bands, one migrating slightly faster than human $\mathrm{B} / \mathrm{B}^{\prime}$ and the other somewhat slower than human D1. This is in good agreement with the known size differences of the corresponding proteins: Human $\mathrm{Sm} \mathrm{B} / \mathrm{B}^{\prime}$ has a molecular mass of $\sim 24 \mathrm{kD}$ (but actually migrates as a $\sim 29-\mathrm{kD}$ protein), whereas the corresponding mass for Drosophila Sm B is $\sim 22$ $\mathrm{kD}$; the human and Drosophila D1 proteins are $\sim 13$ and $\sim 14$ $\mathrm{kD}$ in mass, respectively. Thus, the Y12 antibody, in S2 cells, 

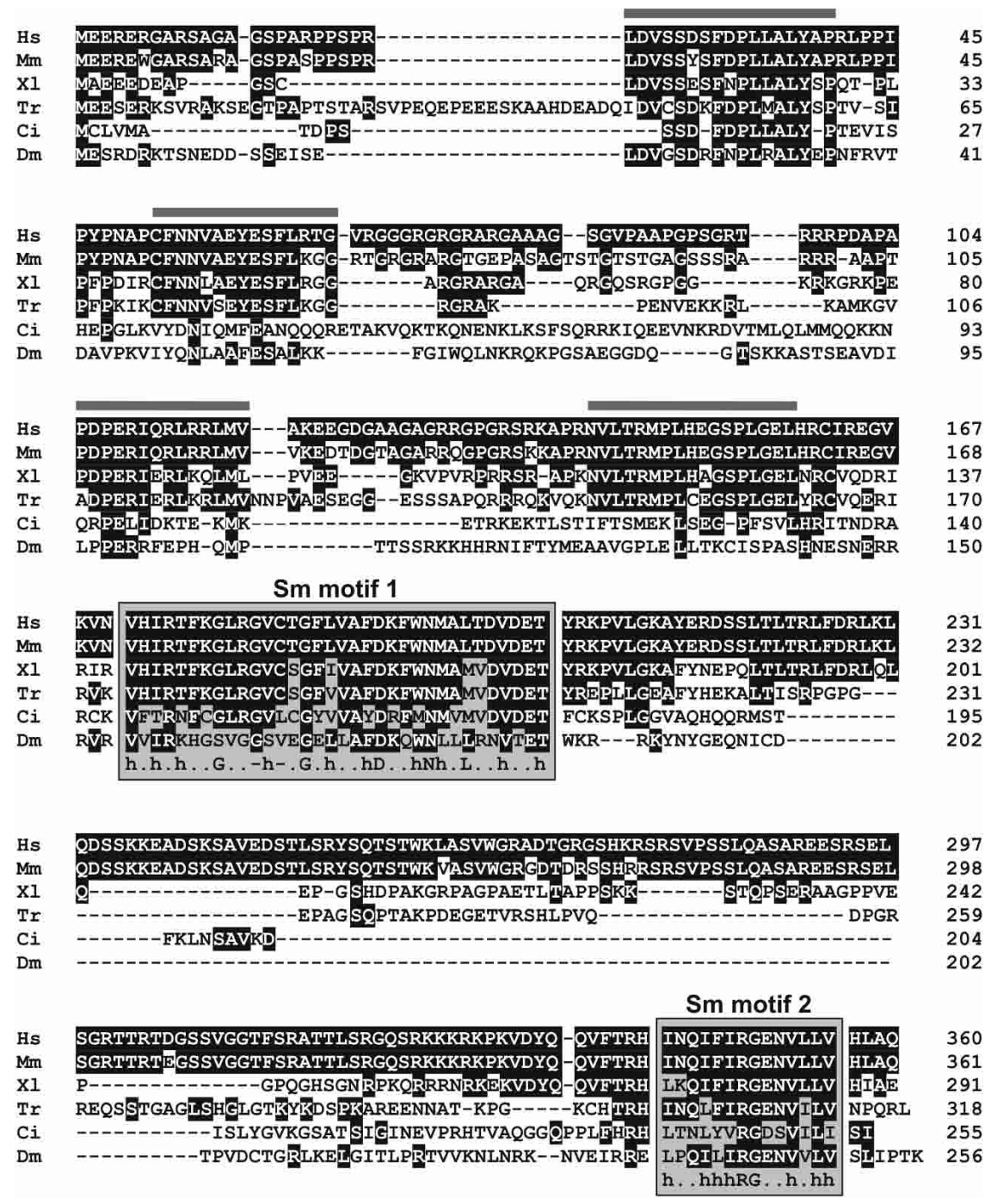

FIGURE 2. Sequence alignment of Lsm11 proteins. Additional sequences, accession numbers, and links are available at our online database (http://www.izb.unibe.ch/res/schuehome/ schuemperli/Lsm11.html). The sequences are from the following species: Hs, Homo sapiens, human (UniGene Hs.187117); Mm, Mus musculus, house mouse (UniGene Mm.45659); Xl, Xenopus laevis (GenBank AF514310, UniGene Xl.13277); Tr, Takefugu rubripes, pufferfish (scaffold 544, positions 60'000-63'000); Ci, Ciona intestinalis, transparent sea squirt (ascidian tunicate; Unigene Cin.4879); and Dm, Drosophila melanogaster (Flybase CG12924). Residues identical to the human protein are shown in inverse print. Dark grey bars indicate conserved sequence patches in the $\mathrm{N}$-terminal region. The conserved Sm motifs 1 and 2 are indicated by shaded boxes. The consensus sequences were adapted from previous sources (Hermann et al. 1995; Achsel et al. 2001). h indicates hydrophobic amino acids.

appears to react primarily with the Drosophila Sm B and D1 proteins.

\section{Drosophila Lsm10 and Lsm11 assemble into complexes that contain $\mathrm{Sm} B$ but are lacking Sm D1 and D2}

Although the above experiments strongly indicated that dLsm10 and dLsm11 are part of a snRNP complex containing at least some of the common Sm proteins, we wanted to obtain more specific information regarding the composition of this complex. In particular, we have shown that mammalian Lsm10 and Lsm11 replace Sm D1 and D2 in the unique U7 Sm core structure (Pillai et al. 2001, 2003). We postulated that, if dLsm10 and dLsm11 are true orthologs of the corresponding mammalian proteins and if the U7 Sm core structure has been conserved between vertebrates and arthropods, the two proteins should also be in a complex containing Sm B but lacking D1 and D2 in Drosophila S2 cells.

This hypothesis was tested by using additional expression constructs encoding Flag-tagged versions of the Drosophila Sm B and D2 ORFs. Flag-dB was well expressed after transfection of S2 cells (Fig. 4A,C,E, input lanes), whereas the expression of Flag-dD2 was low and sometimes barely detectable in wholecell extract (Fig. 4B,D,F, input lanes). More importantly, both Flag- $\mathrm{dB}$ and Flag-dD2 were efficiently precipitated by Y12 antibody, indicating that they were incorporated into snRNPs. In contrast, the anti-dLsm11 antibody precipitated only Flag-dB, not Flag-dD2 (Fig. $4 A, B$, upper panels). Reprobing of the blots with Y12 antibody revealed both Sm B and D1 in the Y12 precipitates, but only Sm B, not D1, in the antidLsm11 precipitates (Fig. 4A,B, lower panels).

Even though these results were in agreement with the above hypothesis, we tried to corroborate them by additional experiments, especially because the lack of an Sm D1 signal in the Y12 immunoblots could also have been due to the generally low recovery of proteins in the anti-dLsm11 immunoprecipitations. Thus, combinations of HA-tagged dLsm10 or dLsm11 with Flag-tagged Sm $\mathrm{dB}$ or $\mathrm{dD} 2$ were cotransfected into S2 cells; whole-cell extracts were subjected to immunoprecipitation with anti-HA antibody and probed with either anti-Flag, anti-HA or Y12 anti-Sm antibody. In cells cotransfected with Flag-dB in combination with either HA-dLsm11 (Fig. 4C) or HAdLsm10 (Fig. 4E), both Flag-dB and the HA-tagged Lsm protein could be revealed in the anti-HA immunoprecipitates. In contrast Flag-dD2 could not be coprecipitated with either HA-dLsm11 (Fig. 4D) or HA-dLsm10 (Fig. 4F), although the corresponding HA-tagged Lsm proteins were 


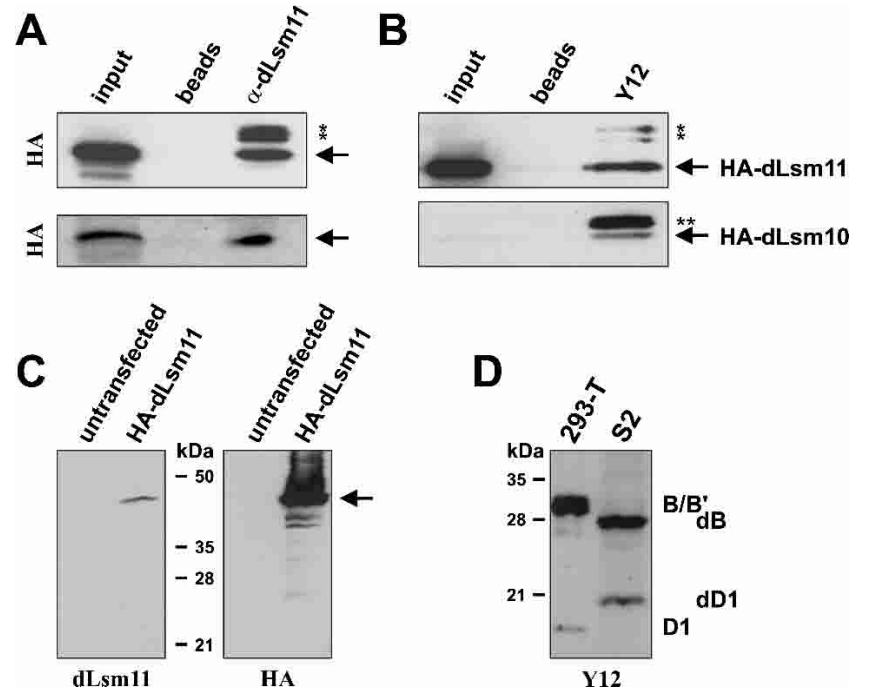

FIGURE 3. In vivo association of Drosophila Lsm10 and Lsm11 with each other and with Sm proteins. (A) Immunoprecipitation by an affinity-purified antibody raised against recombinant GST-tagged dLsm11 ( $\alpha$-dLsm11). Drosophila S2 cells were transiently transfected with expression plasmids encoding HA-tagged dLsm11 (top) or dLsm10 (bottom), and whole-cell extracts were subjected to immunoprecipitation, SDS-PAGE, and Western blotting with anti-HA antibody. Input indicates sample of nuclear extract prior to immunoprecipitation; beads, control precipitation by protein G-Sepharose beads without antibody. Asterisks $\left(^{*}\right)$ indicate bands of slower electrophoretic mobility that react with the anti-HA antibody subsequent to immunoprecipitation; it is not known whether these represent potentially modified HA-dLsm11 protein (the expected reacting band is indicated by an arrow). (B) Immunoprecipitation of nuclear extracts from similar transfections by monoclonal anti-Sm antibody Y12. Lane labels and symbols are used as in A. (C) Reactivity of the anti-dLsm11 antibody used in $A$. Duplicate samples of whole-cell extracts from untransfected Drosophila S2 cells or cells stably expressing HA-dLsm11 were subjected to SDS-PAGE and blotted onto the same filter. The two filter halves were probed with either anti-dLsm11 or anti-HA antibodies as indicated below the panels. Both antibodies react with a protein of identical electrophoretic mobility representing HA-dLsm11 (arrow). (D) Reactivity of the anti-Sm antibody Y12 used in B Nuclear extracts from human 293-T cells and from Drosophila S2 cells were subjected to SDS-PAGE and Western blotting with Y12 antibody. The antibody reacts primarily with human $\mathrm{Sm} B / \mathrm{B}^{\prime}$ and $\mathrm{D} 1$ and with their corresponding Drosophila orthologs.

efficiently precipitated by the anti-HA antibody. This indicated that the complex containing Drosophila Lsm10 and Lsm11 proteins contains Sm B, but not D2, as would be predicted, in analogy with the composition of mammalian U7 snRNPs. Furthermore, reprobing the same blots or blots prepared from identical cotransfection/immunoprecipitation experiments with Y12 antibody revealed the coprecipitation of endogenous Drosophila Sm B, but not D1, by the anti-HA antibody (Fig. 4C-F, bottom panels), indicating that the complex containing Drosophila Lsm10 and Lsm11 proteins does not contain Sm D1. Note, that more Sm dB was precipitated by anti-HA than by anti-dLsm11 antibody (Fig. 4A,B), presumably because of the higher expression of the HA-tagged dLsm10 and dLsm11 proteins and/or be- cause of more efficient immunoprecipitation by the antiHA antibody.

\section{Drosophila Lsm10 and Lsm11 associate with U7 snRNA and histone pre-mRNA in S2 cells}

Having established that dLsm 10 and dLsm 11 show the same pattern of associations with Sm proteins as do their counterparts in the mammalian U7 snRNP, we wanted to analyze if they also associate with Drosophila U7 snRNA in S2 cells. This was facilitated by the recent determination of the Drosophila U7 snRNA sequence (Dominski et al. 2003). We designed primers allowing the detection of Drosophila U7 snRNA by reverse transcription-coupled PCR (RT-PCR). The expected RT-PCR signal of 88 nucleotides was produced by using RNA isolated from Drosophila S2 cell nuclear extract as template (Fig. 5A, lane 1). The RNA
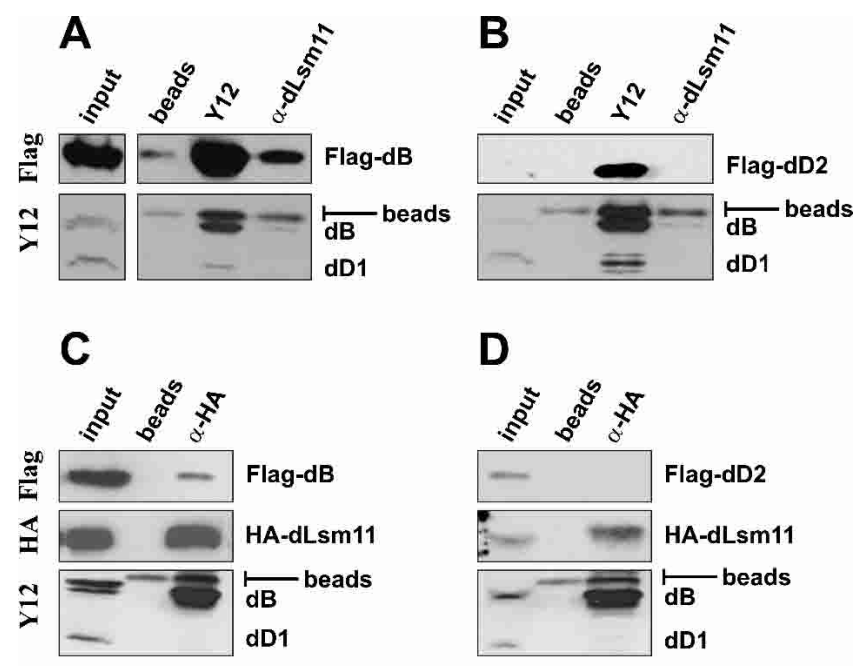

D
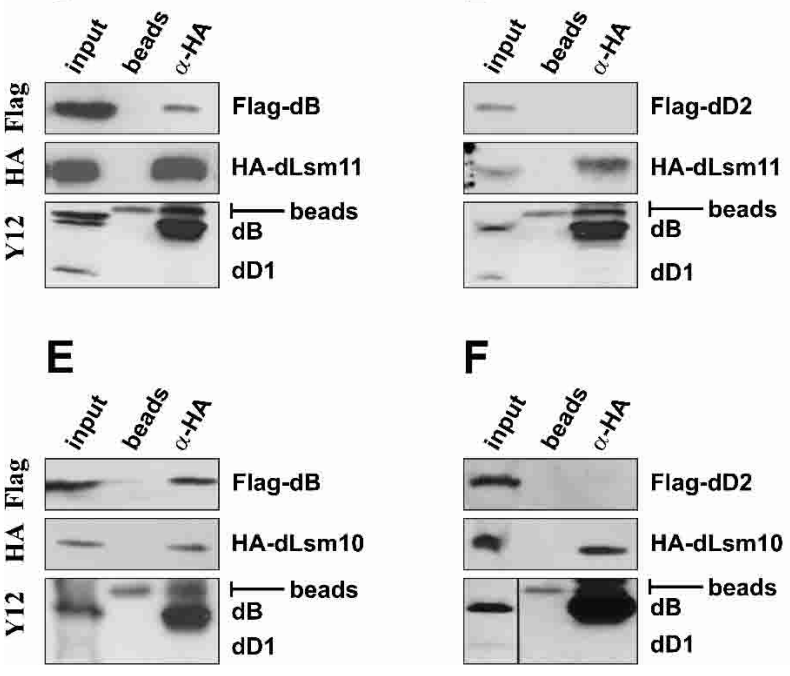

FIGURE 4. In vivo association of Drosophila Lsm10 and Lsm11 with Sm B, but not with Sm D1 and D2. Drosophila S2 cells were transiently transfected with expression plasmids encoding Flag-tagged Sm proteins $\mathrm{dB}(A, C, E)$ or $\mathrm{dD} 2(B, D, F)$ either alone $(A, B)$ or in combination with HA-dLsm11 $(C, D)$ or HA-dLsm10 $(E, F)$. Whole-cell extracts were subjected to immunoprecipitation with the antibodies indicated above the lanes. The samples were subjected to SDS-PAGE, and Western blots were probed by using the antibodies indicated on the left of the figure. Input indicates sample of nuclear extract prior to immunoprecipitation; beads, control precipitation by protein G-Sepharose beads without antibody. A band corresponding to protein $\mathrm{G}$ released from the beads is visible in all the Y12 blots, just above the band specific for Sm dB. The black separation line in the bottom panel of $F$ indicates that the input sample was run in a different position on this gel and had to be remounted. 

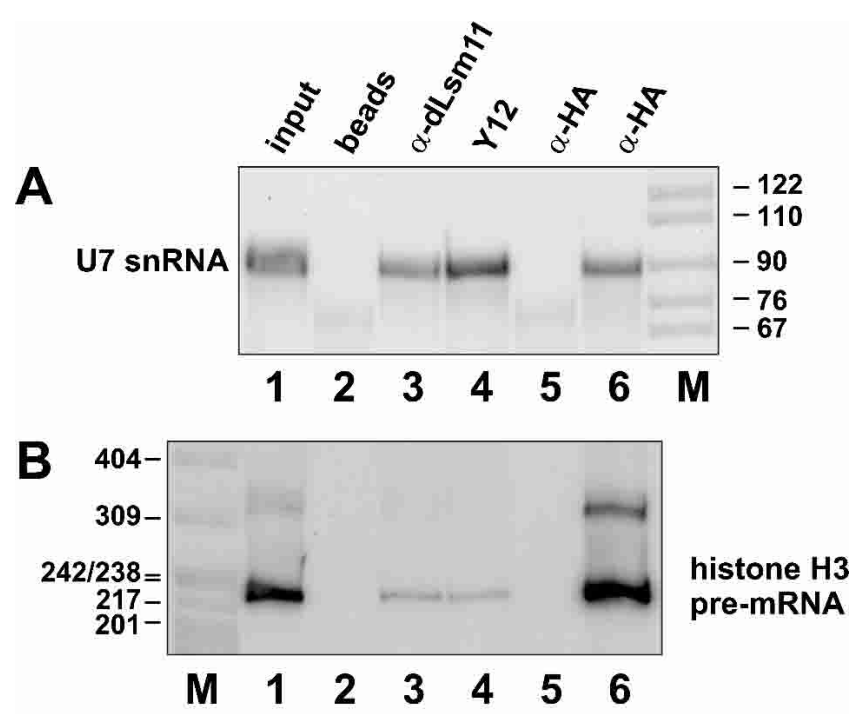

C

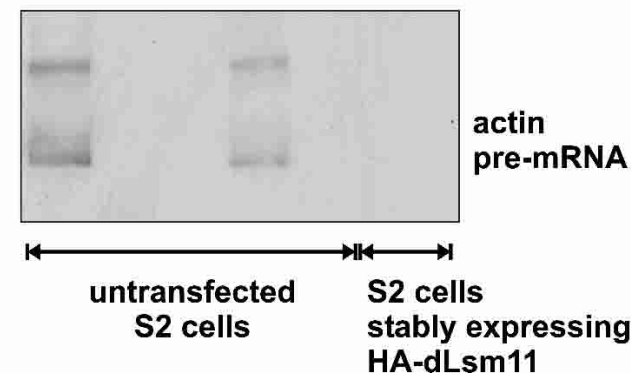

FIGURE 5. Association of Drosophila Lsm11 with Drosophila U7 snRNA and histone H3 pre-mRNA. Nuclear extracts of either untransfected Drosophila S2 cells (lanes 2-5) or S2 cells stably expressing HA-dLsm11 (lane 6) were subjected to precipitation with protein G-Sepharose beads either lacking antibody (lane 2) or coated with the antibodies indicated above each lane. Lane 1, sample of input nuclear extract from untransfected S2 cells. RNA from each sample was extracted and subjected to RT-PCR for Drosophila U7 snRNA $(A)$, histone $\mathrm{H} 3$ pre-mRNA $(B)$, or $\beta$-actin pre-mRNA $(C)$. $\mathrm{M}$ indicates endlabeled HpaII digest of pBR322 used as size marker.

responsible for this band was immunoprecipitated with anti-dLsm11 (lane 3) and Y12 antibodies (lane 4), but it could not be precipitated by protein G-Sepharose beads without antibodies (lane 2). Moreover, in S2 cells stably expressing HA-dLsm11, U7 snRNA could be immunoprecipitated with anti-HA antibody (lane 6), whereas anti-HA precipitation of extract from cells not expressing HAdLsm11 did not coprecipitate U7 snRNA (lane 5). Thus, Drosophila U7 snRNA is associated with Sm proteins and, more specifically, with dLsm11 in vivo.

Next, we analyzed whether precipitating the U7 snRNP with anti-dLsm11 antibody also coprecipitated histone premRNA, as would be expected for a particle involved in histone RNA 3' end processing. Thus, samples of the RNAs already used in Figure 5A were analyzed by RT-PCR assays for histone $\mathrm{H} 3$ pre-mRNA and, as a negative control, for $\beta$-actin pre-mRNA. The results showed that histone $\mathrm{H} 3$ pre-mRNA could be precipitated by both anti-dLsm11 (Fig.
5B, lane 3) and Y12 antibodies (lane 4), but not by protein G-Sepharose beads alone (lane 2). Moreover, similar to U7 snRNA shown above, $\mathrm{H} 3$ pre-mRNA was also enriched by immunoprecipitation with anti-HA antibody from nuclear extracts of S2 cells stably expressing HA-dLsm11 (lane 6). This was due to a specific association of $\mathrm{H} 3$ pre-mRNA with a complex containing HA-dLsm11, because the anti-HA antibody did not precipitate $\mathrm{H} 3$ pre-mRNA from nuclear extract of S2 cells not expressing the HA-tagged protein (lane 5). As expected and in contrast to $\mathrm{H} 3$ pre-mRNA, $\beta$-actin pre-mRNA could only be detected in the input nuclear extract and in the Y12 immunoprecipitation (presumably due to its association with spliceosomal snRNPs), but not in any of the other immunoprecipitates (Fig. 5C). These experiments indicated that the complex containing Drosophila Lsm11 protein is also, albeit temporarily, associated with histone $\mathrm{H} 3$ pre-mRNA.

\section{Assembly of Drosophila Lsm10 and Lsm11 into mammalian U7 snRNPs}

As the above experiments indicated that the composition of the U7 Sm core is conserved between vertebrates and arthropods, we tested whether dLsm 10 and dLsm 11 could be assembled into U7 snRNPs in mammalian cells and whether, by doing so, they could discriminate between U7 and U1 snRNA. For this purpose, we transiently co-expressed HA-dLsm10 and HA-dLsm11 under the control of the human cytomegalovirus promoter in human 293-T cells. U7 or U1 snRNPs were precipitated from nuclear extracts by using biotinylated oligonucleotides complementary to the $5^{\prime}$ ends of the corresponding snRNAs. The HAtagged proteins were then detected in the precipitates by Western blotting with anti-HA antibody. Indeed, both HAdLsm10 and HA-dLsm11 associated with U7 snRNA, but similar amounts of the two proteins were also precipitated by the anti-U1 oligonucleotide (Fig. 6, upper panel). However, when comparing these band intensities, one must consider that the cells contain $\sim 100$ times more U1 than U7 snRNPs (Grimm et al. 1993) and that different amounts of snRNPs will therefore be precipitated by the two antisense oligonucleotides. This was confirmed by reprobing the Western blot with Y12 anti-Sm antibody, which detects the $\mathrm{Sm} \mathrm{B} / \mathrm{B}^{\prime}$ protein present in both the $\mathrm{U} 7$ and $\mathrm{U} 1$ snRNPs

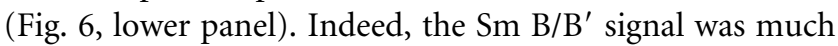
stronger in the precipitate obtained with U1 oligonucleotide. This implied that dLsm10 and dLsm11 preferentially bound to U7 snRNA. Taken together, these results indicated that the protein-protein and protein-RNA interactions involved in the assembly of the U7 Sm core must be evolutionarily conserved between arthropods and vertebrates.

\section{DISCUSSION}

The central role of the U7 snRNP for histone RNA $3^{\prime}$ end processing and its importance for studying Sm core assem- 


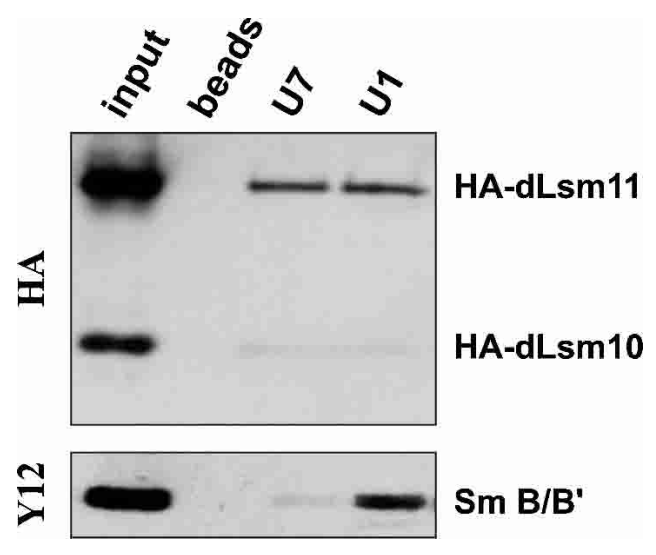

FIGURE 6. Association of Drosophila Lsm10 and Lsm11 with U7 snRNA in mammalian cells. Human 293-T cells were cotransfected with plasmids encoding HA-dLsm10 and HA-dLsm11. Nuclear extracts were subjected to precipitation with magnetic streptavidin beads coated with biotinylated oligonucleotides complementary to human U7 or U1 snRNAs as indicated. The pellets were subjected to SDSPAGE, and a Western blot was probed with anti-HA or Y12 antibody (revealing $\mathrm{Sm} \mathrm{B} / \mathrm{B}^{\prime}$ ), as indicated on the left of the figure. Input indicates sample of original nuclear extract; beads, precipitation with beads not coated with oligonucleotide.

bly, nuclear import of snRNPs, as well as Cajal body formation and function (see Introduction) motivated us to characterize this particle in Drosophila, an organism that is amenable to various types of investigation (classical and reverse genetics, RNAi, biochemistry, developmental studies). Moreover, through the study of evolutionarily distant orthologs, we hoped to identify conserved amino acid residues that may provide important cues for more detailed functional studies. Finally, as mentioned in the Introduction, evolutionary aspects of histone RNA processing were also of considerable interest in their own right. The fact that orthologs of Lsm10 and Lsm11, ZFP100 and, until recently, U7 snRNA had not been identified in lower invertebrates, allowed the alternative hypothesis that histone RNA processing, despite the presence of the conserved hairpin and HBP/SLBP, might operate by a mechanism independent of a U7 snRNP in these organisms. Initial difficulties to identify Lsm10 orthologs in invertebrates (Pillai et al. 2001) also raised the possibility that only Lsm 11 but not Lsm10 might be present.

Our identification of putative Lsm10 and Lsm11 sequences in various invertebrates (Figs. 1,2) and the characterization of the corresponding Drosophila proteins as true orthologs of their vertebrate counterparts contradict both of these alternative views. In Drosophila S2 cells, $\mathrm{dLsm} 10$ and dLsm11 associate with each other and with Sm B, as well as with Drosophila U7 snRNA (Figs. 3-5). Conversely, Sm dD1 and $\mathrm{dD} 2$ are not found associated with either dLsm10 or dLsm11. This pattern of associations is identical to the one found in vertebrates. Thus, although we have not analyzed the other Sm proteins, we postulate that the composition of the U7 Sm core structure is identical in arthropods and in vertebrates. Moreover, despite the fact that a Drosophila ZFP100 ortholog has not yet been identified, the basic constituents and hence also the mechanism of histone RNA processing appear to be the same in both types of organisms. Extrapolating from these findings, it seems likely that the key elements of the histone RNA 3' processing machinery will also be conserved in the other metazoans and in the additional organisms that have nonpolydenylated histone mRNAs ending in the conserved hairpin (Wittop Koning and Schümperli 1994) or for which a putative HBP/SLBP ortholog can be found in sequence databases.

This similarity in the composition of the U7 snRNP and the histone RNA processing machinery contrasts with a high degree of primary sequence divergence of individual components. The recently characterized Drosophila U7 snRNA (Dominski et al. 2003) is so different from its known vertebrate and sea urchin counterparts that it could not be discovered by a bioinformatic approach. The same is true for the Lsm10 protein, for which we initially could not find any invertebrate orthologs (Pillai et al. 2001). The sequences of the potential tunicate and arthropod orthologs are so different that only the biochemical experiments presented in Figures 3-5 could confirm the identification of the Drosophila protein as Lsm10.

The situation is somewhat different for the large Lsm11 protein (Fig. 2). The Drosophila and Ciona orthologs were quite readily discovered by bioinformatic searching. Apart from the characteristic Sm motifs, two prominent features of Lsm11 are conserved in all potential orthologs: The first $\mathrm{Sm}$ motif is preceded by a large $\mathrm{N}$-terminal extension, and the two Sm motifs are separated by a long spacer. This spacer is not conserved in primary sequence, but the fact that it is present in all putative orthologs suggests that it may perhaps play a role that is not sequence specific. Consistent with the possibilities both of a lack of function or of a sequence-unspecific function, we have previously shown that more than half of this spacer can be deleted from murine Lsm11 without affecting either U7 snRNP assembly or histone RNA processing (Pillai et al. 2003). In contrast, the phylogenetic comparison with invertebrate sequences reveals that the $\mathrm{N}$ terminus of Lsm11 contains four conserved sequence patches. Secondary structure modeling by the PHD program suite (Rost and Sander 1994, 2000) indicates a high propensity of these sequences for $\alpha$-helix formation, whereas the remainder of the $\mathrm{N}$ terminus does not contain any predicted secondary structure elements. It goes without saying that these conserved sequence patches are primary candidates for being functionally involved in histone RNA processing and in the binding of ZFP100 or of additional, yet unknown processing factors.

The coprecipitation of Drosophila U7 snRNA and histone H3 pre-mRNA with anti-dLsm11 antibody (Fig. 5) confirms that the characterized particle indeed is the Drosophila U7 snRNP. Moreover, Drosophila U7 snRNA is also co- 
precipitated by anti-HA antibody in S2 cells stably expressing HA-dLsm11, but not in S2 cells that do not express any HA-tagged protein. This proves that HA-dLsm11 indeed gets incorporated into U7 snRNPs and validates the results from the association studies carried out with tagged proteins. Although we do not yet know if the U7 snRNPs containing HA-dLsm11 are functional in histone RNA processing, they are able to interact with histone $\mathrm{H} 3$ premRNA, just like U7 snRNPs containing the endogenous, untagged dLsm11, as judged by the coprecipitation of $\mathrm{H} 3$ pre-mRNA (Fig. 5). Thus the tagged protein may in the future be useful for functional complementation studies, either in flies carrying a mutation in the dLsm 11 gene or in S2 cells in which the dLsm11 gene has been silenced by RNA interference.

The ability of HA-dLsm10 and HA-dLsm11 to get incorporated into U7 snRNPs in mammalian cells (Fig. 6) indicates that despite considerable differences at the primary sequence level, the protein-protein and protein-RNA interactions involved in U7 snRNP assembly must be evolutionarily conserved. However, initial experiments in which the two Drosophila Lsm proteins were transfected individually resulted in a less efficient incorporation into U7 snRNPs and, for HA-dLsm10, also in a poorer expression (data not shown). As Sm core assembly proceeds through specific Sm protein heterodimers and heterotrimers (Raker et al. 1996, 1999) and as Lsm10 and Lsm11 are presumably part of the same heterodimer (Pillai et al. 2003), the poorer performance of individual Drosophila Lsm proteins is most likely due to the fact that they must form an Lsm10/11 heterodimer with a partner from a different species.

Although the cotransfected HA-dLsm 10 and HA-dLsm11 showed a preference for U7 over U1 snRNA in mammalian cells (Fig. 6), it must be noted that this discrimination was less pronounced than when mammalian Lsm10 or Lsm11 proteins were previously tested in the same kind of assay (Pillai et al. 2001, 2003). The reason for this reduced selectivity is unknown, but this phenomenon deserves further investigation, as such studies may contribute to understanding how the U7-specific and spliceosomal Sm core structures are specified. Moreover, it will be interesting to see if U7 snRNPs formed with Drosophila Lsm10/11 in a vertebrate context are functional in histone RNA $3^{\prime}$ processing.

In conclusion, these studies have revealed that the special architecture of the U7 snRNP is evolutionarily conserved, and have opened the possibility to study the assembly and function of this interesting particle in the Drosophila system that is equally amenable to classical and reverse genetics as well as to biochemical investigation.

\section{MATERIALS AND METHODS}

\section{Plasmids}

Original clones were obtained through the Berkeley Drosophila Genome Project Database (BDGP). The dLsm11 (EST BG640322),
dLsm10 (genomic bacmid BAC R13J10), dSmB (EST LD14049), and dSmD2 (EST LD03002) ORFs were amplified by PCR and subcloned for expression in Drosophila cells into modified pMTv/A expression vectors (Invitrogen). These pMTv-HA and pMTv-Flag vectors contained N-terminal HA (MAYPYDVPDY ASLE) and Flag (DYKDDDDK) epitope tag-encoding sequences, respectively, introduced as annealed DNA oligonucleotides.

For expression in mammalian cells as N-terminal HA tag fusions, the dLsm10 and dLsm11 ORFs were introduced into the pcDNA3-HA vector (Pillai et al. 2001). For expression in Escherichia coli as N-terminal GST fusion, the dLsm11 ORF was subcloned into pGex4T-3 (Pharmacia). All clones were verified by DNA sequencing. Details of the constructs are available on request.

\section{Antibodies}

To produce the antibody referred to as anti-dLsm11, insoluble GST-dLsm 11 protein was recovered from the pellet fraction of the producing Escherichia coli strain BL21 (Pharmacia) and resolved by SDS-PAGE, and a gel fragment mixed with adjuvants was used for immunization of rabbits. Antibodies were affinity-purified by overnight incubation with recombinant proteins blotted on a nitrocellulose membrane, essentially as described (Pillai et al. 2001).

Additionally, monoclonal antibodies against $\mathrm{Sm}$ proteins $\mathrm{B} / \mathrm{B}^{\prime}$, D1, and D3 (Y12; Lerner et al. 1981) or against the HA tag (BABCO) were used. The HA and Flag epitope tags were detected in Western blots using anti-HA (Roche) and anti-Flag (Sigma) antibodies directly coupled to horseradish peroxidase.

\section{Cell culture and transfections}

Human 293-T cells were cultured as described (Pillai et al. 2001). For transfection, they were grown in $10-\mathrm{cm}$ dishes to $50 \%-60 \%$ confluency and transfected with a mixture of $5 \mu \mathrm{g}$ each of pcDNA3-HA-dLsm10 and pcDNA3-HA-dLsm11 using Lipofectamine (Invitrogen). Cells were harvested $48 \mathrm{~h}$ after transfection.

Drosophila Schneider S2 cells were cultured in Schneider's Drosophila medium with L-glutamine (AMIMED), supplemented with $10 \%$ heat-inactivated fetal calf serum, $100 \mathrm{U} / \mathrm{mL}$ penicillin $\mathrm{G}$, and $100 \mu \mathrm{g} / \mathrm{mL}$ streptomycin (AMIMED) at $26^{\circ} \mathrm{C}$ (without $\mathrm{CO}_{2}$ ). For transient transfection, $10^{6}$ cells were seeded in $10-\mathrm{cm}$ dishes, grown to $\sim 70 \%$ confluency, and transfected with $10 \mu \mathrm{g}$ (or mixtures of $2 \times 5 \mu \mathrm{g}$ ) of pMTv-derived plasmids by using CellFectin (Invitrogen). Expression of proteins from the metallothionein promoter was induced at day 2 by the addition of $500 \mu \mathrm{M}$ copper sulfate to the medium, and cultures were continued for another $4 \mathrm{~d}$.

For stable transfection, S2 cells were cotransfected with pMTvHA-dLsm 11 and the pCoHygro selection vector (Invitrogen) in a 19:1 ratio ( $4 \mu \mathrm{g} /$ well of a six-well plate), according to the manufacturer's instructions. Five days later, the cells were harvested and resuspended in Schneider's medium, supplemented as above but additionally containing $400 \mu \mathrm{g} / \mathrm{mL}$ hygromycin $\mathrm{B}$, and grown for 3 to 4 weeks. Stably transfected S2 cells were split when the cell density reached between 2 and $6 \times 10^{6}$ cells/mL (Hill et al. 2001).

\section{Extract preparation and protein interaction studies}

The preparation of small-scale nuclear or whole-cell extracts, immunoprecipitations, and precipitations with biotinylated oligo- 
TABLE 1. Primers and amplicon lengths for RT-PCR

\begin{tabular}{|c|c|}
\hline U7 snRNA & $\begin{array}{ll}\text { dU7-5': } & 5^{\prime} \text {-GACAACTCATTGAAAATTTTTATTCTCTTTG } \\
\text { dU7-3': } & 5^{\prime} \text {-TATAATTAGAACGGGAACACTCAATGCCTAG } \\
& \text { (sequences corresponding to dU7 snRNA are underlined) } \\
\text { amplicon length: } 88 \mathrm{bp}\end{array}$ \\
\hline $\begin{array}{l}\text { Histone H3 } \\
\text { pre-mRNA }\end{array}$ & $\begin{array}{ll}\text { dH3-5': } & 5^{\prime} \text {-GCGAAGCCTACCTGGTTGGTCTCTTCGAAG } \\
\text { dH3-3': } & 5^{\prime} \text {-CICTCTAGAAACAGAAAATTTTATCTCATTGAATCTGG } \\
& \text { (sequences corresponding to the histone } H 3 \text { gene are underlined) } \\
\text { amplicon length: } 230 \mathrm{bp}\end{array}$ \\
\hline $\begin{array}{l}\beta \text {-Actin } \\
\text { pre-mRNA }\end{array}$ & $\begin{array}{ll}\text { dAct-5': } & 5^{\prime} \text {-CCAGCGTATTAATATAATTAATTTATTCCAC } \\
\text { dAct-3' } & 5^{\prime} \text {-'TTCTTTATCTTGATGTCCTTGTCATGCC } \\
& \text { (sequences corresponding to the } \beta \text {-actin gene are underlined) } \\
\text { amplicon length: } 234 \mathrm{bp}\end{array}$ \\
\hline
\end{tabular}

Bond, U.M., Yario, T.A., and Steitz, J.A. 1991. Multiple processing-defective mutations in a mammalian histone premessenger RNA are suppressed by compensatory changes in U7 RNA both in vivo and in vitro. Genes \& Dev. 5: 1709-1722.

Brahms, H., Raymackers, J., Union, A., de Keyser, F., Meheus, L., and Lührmann, R. 2000. The C-terminal RG dipeptide repeats of the spliceosomal Sm proteins D1 and D3 contain symmetrical dimethylarginines, which form a major B-cell epitope for anti-Sm autoantibodies. J. Biol. Chem. 275: 1712217129.

Brahms, H., Meheus, L., de Brabandere, V., Fischer, U., and Lührmann, R. 2001. Symmetrical dimethylation of arginine residues in spliceosomal $\mathrm{Sm}$ protein $\mathrm{B} / \mathrm{B}^{\prime}$ and the Sm-like protein LSm4, and their interaction nucleotides complementary to the $5^{\prime}$ ends of U7 or U1 snRNA were performed as described (Pillai et al. 2001).

Proteins were resolved on 12\% high-TEMED SDS-polyacrylamide gels (Will et al. 1994). Western blots were probed with Y12 anti-Sm (Lerner et al. 1981), anti-HA-HRP, or anti-Flag-HRP antibodies and developed by the ECL method (Amersham).

\section{Coprecipitation and detection of Drosophila U7 snRNA as well as histone $\mathrm{H} 3$ and $\beta$-actin pre-mRNAs}

Large scale nuclear extracts from Drosophila S2 cells were prepared as described. Total RNAs were isolated from immunoprecipitates obtained with different antibodies (anti-dLsm11, Y12, or anti-HA) from $1 \mathrm{~mL}$ samples of S2 wild-type nuclear extract (large scale; Dignam et al. 1983; Pillai et al. 2001) or from $400 \mu \mathrm{L}$ of nuclear extract from the S2 cell line stably expressing HA-dLsm11 (small scale; Pillai et al. 2001). The RNAs were extracted at $65^{\circ} \mathrm{C}$ with a buffer containing $10 \mathrm{mM}$ Tris- $\mathrm{HCl}$ ( $\mathrm{pH} 8$ ), 5 mM EDTA, $0.5 \%$ SDS, $1 \mathrm{mg}$ proteinase $\mathrm{K}$, and $4 \mu \mathrm{g}$ glycogen.

Drosophila U7 snRNA, histone $\mathrm{H} 3$ pre-mRNA, and $\beta$-actin premRNA were amplified by RT-PCR using the rTth kit (Perkin Elmer) and $\alpha-32 \mathrm{P}-\mathrm{dCTP}$, essentially as described (Suter et al. 1999). The primers and conditions used are listed in Table 1. RT and PCR conditions were selected for each primer pair and are available on request.

\section{ACKNOWLEDGMENTS}

We thank O. Mühlemann and B. Suter for critical comments, as well as K. Schranz and A. Gruber for technical help. This work was supported by the State of Bern and by Swiss National Science Foundation grant 31-65225.01 to D.S.

The publication costs of this article were defrayed in part by payment of page charges. This article must therefore be hereby marked "advertisement" in accordance with 18 USC section 1734 solely to indicate this fact.

Received July 28, 2003; accepted September 4, 2003.

\section{REFERENCES}

Achsel, T., Stark, H., and Lührmann, R. 2001. The Sm domain is an ancient RNA-binding motif with oligo(U) specificity. Proc. Natl. Acad. Sci. 98: 3685-3689. with the SMN protein. RNA 7: 1531-1542.

Dignam, J.D., Lebovitz, R.M., and Roeder, R.G. 1983. Accurate transcription initiation by RNA polymerase II in a soluble extract from isolated mammalian nuclei. Nucl. Acids Res. 11: 1475-1489.

Dominski, Z. and Marzluff, W.F. 1999. Formation of the 3' end of histone mRNA. Gene 239: 1-14.

Dominski, Z., Zheng, L.X., Sanchez, R., and Marzluff, W.F. 1999. Stem-loop binding protein facilitates $3^{\prime}$-end formation by stabilizing U7 snRNP binding to histone pre-mRNA. Mol. Cell. Biol. 19: $3561-3570$.

Dominski, Z., Erkmann, J.A., Yang, X., Sanchez, R., and Marzluff, W.F. 2002a. A novel zinc finger protein is associated with U7 snRNP and interacts with the stem-loop binding protein in the histone pre-mRNP to stimulate $3^{\prime}$-end processing. Genes \& Dev. 16: $58-71$.

Dominski, Z., Yang, X.C., Raska, C.S., Santiago, C., Borchers, C.H., Duronio, R.J., and Marzluff, W.F. 2002b. 3' end processing of Drosophila melanogaster histone pre-mRNAs: Requirement for phosphorylated Drosophila stem-loop binding protein and coevolution of the histone pre-mRNA processing system. Mol. Cell. Biol. 22: 6648-6660.

Dominski, Z., Yang, X.C., Purdy, M., and Marzluff, W.F. 2003. Cloning and characterization of the Drosophila U7 small nuclear RNA. Proc. Natl. Acad. Sci. 100: 9422-9427.

Frey, M.R. and Matera, A.G. 1995. Coiled bodies contain U7 small nuclear RNA and associate with specific DNA sequences in inter-

Grimm, C., Stefanovic, B., and Schümperli, D. 1993. The low abundance of U7 snRNA is partly determined by its Sm binding site. EMBO J. 12: 1229-1238.

Harris, M.E., Böhni, R., Schneiderman, M.H., Ramamurthy, histone mRNA in the unperturbed cell cycle: Evidence suggesting control at two posttranscriptional steps. Mol. Cell. Biol. 11: 24162424.

Hermann, H., Fabrizio, P., Raker, V.A., Foulaki, K., Hornig, H., Brahms, H., and Lührmann, R. 1995. snRNP Sm proteins share two evolutionarily conserved sequence motifs which are involved in Sm protein-protein interactions. EMBO J. 14: 2076-2088.

Hill, R.M., Brennan, S.O., and Birch, N.P. 2001. Expression, purification, and functional characterization of the serine protease inhibitor neuroserpin expressed in Drosophila S2 cells. Protein Expr. Purif. 22: 406-413.

Kambach, C., Walke, S., and Nagai, K. 1999. Structure and assembly of the spliceosomal small nuclear ribonucleoprotein particles. Curr. Opin. Struct. Biol. 9: 222-230.

Lehmeier, T., Foulaki, K., and Lührmann, R. 1990. Evidence for three distinct D proteins, which react differentially with anti-Sm autoantibodies, in the cores of the major snRNPs U1, U2, U4/U6 and U5. Nucl. Acids Res. 18: 6475-6484. phase human cells. Proc. Natl. Acad. Sci. 92: 5915-5919. L., Schümperli, D., and Marzluff, W.F. 1991. Regulation of 
Lerner, E.A., Lerner, M.R., Janeway, C.A., and Steitz, J.A. 1981. Monoclonal antibodies to nucleic acid-containing cellular constituents: Probes for molecular biology and autoimmune disease. Proc. Natl. Acad. Sci. 78: 2737-2741.

Martin, F., Schaller, A., Eglite, S., Schümperli, D., and Müller, B. 1997. The gene for histone RNA hairpin binding protein is located on human chromosome 4 and encodes a novel type of RNA binding protein. EMBO J. 15: 769-778.

Marzluff, W.F. and Duronio, R.J. 2002. Histone mRNA expression: Multiple levels of cell cycle regulation and important developmental consequences. Curr. Opin. Cell. Biol. 14: 692-699.

Müller, B. and Schümperli, D. 1997. The U7 snRNP and the hairpin binding protein: Key players in histone mRNA metabolism. Semin. Cell Dev. Biol. 8: 567-576.

Pillai, R.S., Will, C.L., Lührmann, R., Schümperli, D., and Müller, B. 2001. Purified U7 snRNPs lack the Sm proteins D1 and D2 but contain Lsm10, a new $14 \mathrm{kDa} S \mathrm{Sm}$ D1-like protein. EMBO J. 20: $5470-5479$.

Pillai, R.S., Grimmler, M., Meister, G., Will, C.L., Lührmann, R., Fischer, U., and Schümperli, D. 2003. Unique Sm core structure of U7 snRNPs: Assembly by a specialized SMN complex and role of a new component, Lsm11, in histone RNA processing. Genes \& Dev. 17: 2321-2333.

Raker, V.A., Plessel, G., and Lührmann, R. 1996. The snRNP core assembly pathway: Identification of stable core protein heteromeric complexes and an snRNP subcore particle in vitro. EMBO J. 15: 2256-2269.

Raker, V.A., Hartmuth, K., Kastner, B., and Lührmann, R. 1999. Spliceosomal U snRNP core assembly: Sm proteins assemble onto an Sm site RNA nonanucleotide in a specific and thermodynamically stable manner. Mol. Cell. Biol. 19: 6554-6565.

Rost, B. and Sander, C. 1994. Combining evolutionary information and neural networks to predict protein secondary structure. Proteins 19: 55-72.

. 2000. Third generation prediction of secondary structures. Methods Mol. Biol. 143: 71-95.

Scharl, E.C. and Steitz, J.A. 1994. The site of $3^{\prime}$ end formation of histone messenger RNA is a fixed distance from the downstream element recognized by the U7 snRNP. EMBO J. 13: 2432-2440.
1996. Length suppression in histone messenger RNA 3'-end maturation: Processing defects of insertion mutant premessenger RNAs can be compensated by insertions into the U7 small nuclear RNA. Proc. Natl. Acad. Sci. 93: 14659-14664.

Schaufele, F., Gilmartin, G.M., Bannwarth, W., and Birnstiel, M.L. 1986. Compensatory mutations suggest that base-pairing with a small nuclear RNA is required to form the $3^{\prime}$ end of $\mathrm{H} 3$ messenger RNA. Nature 323: 777-781.

Spycher, C., Streit, A., Stefanovic, B., Albrecht, D., Wittop, K., and Schümperli, D. 1994. 3' End processing of mouse histone premRNA: Evidence for additional base-pairing between U7 snRNA and pre-mRNA. Nucl. Acids Res. 22: 4023-4030.

Streit, A., Wittop Koning, T.H., Soldati, D., Melin, L., and Schümperli, D. 1993. Variable effects of the conserved RNA hairpin element upon $3^{\prime}$ end processing of histone pre-mRNA in vitro. Nucl. Acids Res. 21: 1569-1575.

Suter, D., Tomasini, R., Reber, U., Gorman, L., Kole, R., and Schümperli, D. 1999. Double-target antisense U7 snRNAs promote efficient skipping of an aberrant exon in three human $\beta$-thalassemic mutations. Hum. Mol. Genet. 8: 2415-2423.

Vasserot, A.P., Schaufele, F.J., and Birnstiel, M.L. 1989. Conserved terminal hairpin sequences of histone mRNA precursors are not involved in duplex formation with the U7 RNA but act as a target site for a distinct processing factor. Proc. Natl. Acad. Sci. 86: 43454349.

Wang, Z.F., Whitfield, M.L., Ingledue, T.C., Dominski, Z., and Marzluff, W.F. 1996. The protein that binds the $3^{\prime}$ end of histone mRNA: A novel RNA-binding protein required for histone premRNA processing. Genes \& Dev. 10: 3028-3040.

Will, C.L., Kastner, B., and Lührmann, R. 1994. Analysis of ribonucleoprotein interactions. In RNA processing, vol. 1 (eds. S.J. Higgins and B.D. Hames), pp. 141-177. Oxford University Press, Oxford, UK.

Wittop Koning, T.H. and Schümperli, D. 1994. RNAs and ribonucleoproteins in recognition and catalysis. Eur. J. Biochem. 219: 2542.

Wu, C.H. and Gall, J.G. 1993. U7 small nuclear RNA in C snurposomes of the Xenopus germinal vesicle. Proc. Natl. Acad. Sci. 90: 6257-6259. 

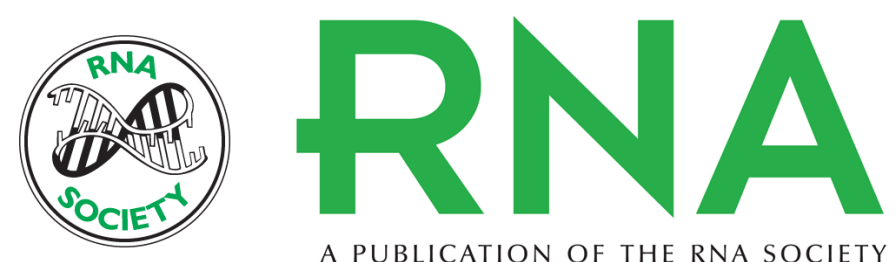

A PUBLICATION OF THE RNA SOCIETY

\section{Evolutionary conservation of the U7 small nuclear ribonucleoprotein in Drosophila melanogaster}

TELDJA N. AZZOUZ and DANIEL SCHÜMPERLI

RNA 2003 9: 1532-1541

References This article cites 37 articles, 18 of which can be accessed free at: http://rnajournal.cshlp.org/content/9/12/1532.full.html\#ref-list-1

\section{License}

Email Alerting Receive free email alerts when new articles cite this article - sign up in the box at the Service top right corner of the article or click here. 